University of Nebraska - Lincoln

DigitalCommons@University of Nebraska - Lincoln

Publications, Agencies and Staff of the U.S.

Department of Commerce

U.S. Department of Commerce

2010

Hierarchical Bayesian Spatial Models for Multispecies

Conservation Planning and Monitoring

\author{
Carlos Carroll \\ Klamath Center for Conservation Research \\ Devin S. Johnson \\ NOAA \\ Jeffrey R. Dunk \\ Humboldt State University \\ William J. Zielinski \\ USDA Forest Service
}

Follow this and additional works at: https://digitalcommons.unl.edu/usdeptcommercepub

Part of the Environmental Sciences Commons

Carroll, Carlos; Johnson, Devin S.; Dunk, Jeffrey R.; and Zielinski, William J., "Hierarchical Bayesian Spatial Models for Multispecies Conservation Planning and Monitoring" (2010). Publications, Agencies and Staff of the U.S. Department of Commerce. 249.

https://digitalcommons.unl.edu/usdeptcommercepub/249

This Article is brought to you for free and open access by the U.S. Department of Commerce at DigitalCommons@University of Nebraska - Lincoln. It has been accepted for inclusion in Publications, Agencies and Staff of the U.S. Department of Commerce by an authorized administrator of DigitalCommons@University of Nebraska - Lincoln. 


\title{
Hierarchical Bayesian Spatial Models for Multispecies Conservation Planning and Monitoring
}

\author{
CARLOS CARROLL, ${ }^{*}$ DEVIN S. JOHNSON, $†$ JEFFREY R. DUNK, $§ \S$ AND WILLIAM J. ZIELINSKI $\S$ \\ *Klamath Center for Conservation Research, Orleans, CA 95556, U.S.A., email carlos@klamathconservation.org \\ †National Marine Mammal Laboratory, Alaska Fisheries Science Center, NOAA, 7600 Sand Point Way N.E., F/AKC3, Seattle, WA \\ 98115, U.S.A. \\ ‡Department of Environmental and Natural Resource Sciences, Humboldt State University, Arcata, California 95521, U.S.A. \\ $\S$ Redwood Sciences Laboratory, Pacific Southwest Research Station, USDA Forest Service, 1700 Bayview Drive, Arcata, California \\ 95521, U.S.A.
}

\begin{abstract}
Biologists who develop and apply habitat models are often familiar with the statistical challenges posed by their data's spatial structure but are unsure of whether the use of complex spatial models will increase the utility of model results in planning. We compared the relative performance of nonspatial and bierarchical Bayesian spatial models for three vertebrate and invertebrate taxa of conservation concern (Church's sideband snails [Monadenia churchi], red tree voles [Arborimus longicaudus], and Pacific fishers [Martes pennanti pacifica]) that provide examples of a range of distributional extents and dispersal abilities. We used presence-absence data derived from regional monitoring programs to develop models with both landscape and site-level environmental covariates. We used Markov chain Monte Carlo algorithms and a conditional autoregressive or intrinsic conditional autoregressive model framework to fit spatial models. The fit of Bayesian spatial models was between 35 and 55\% better than the fit of nonspatial analogue models. Bayesian spatial models outperformed analogous models developed with maximum entropy (Maxent) methods. Although the best spatial and nonspatial models included similar environmental variables, spatial models provided estimates of residual spatial effects that suggested how ecological processes might structure distribution patterns. Spatial models built from presence-absence data improved fit most for localized endemic species with ranges constrained by poorly known biogeographic factors and for widely distributed species suspected to be strongly affected by unmeasured environmental variables or population processes. By treating spatial effects as a variable of interest rather than a nuisance, hierarchical Bayesian spatial models, especially when they are based on a common broad-scale spatial lattice (here the national Forest Inventory and Analysis grid of $24 \mathrm{~km}^{2}$ bexagons), can increase the relevance of habitat models to multispecies conservation planning.
\end{abstract}

Keywords: conditional autoregressive, focal species, hierarchical Bayesian model, Martes pennanti, Northwest Forest Plan, spatial autocorrelation, spatial autoregressive model, spatial dependence, species distribution model

Modelos Espaciales Bayesianos Jerárquicos para la Planificación y Monitoreo de la Conservación de Múltiples Especies

Resumen: Los biólogos que desarrollan y aplican modelos de bábitat a menudo están familiarizados con los retos estadísticos planteados por la estructura espacial de sus datos pero no están seguros sí el uso de modelos espaciales complejos incrementará la utilidad de los resultados del modelo para la planificación.Comparamos el funcionamiento relativo de modelos no espaciales y espaciales Bayesianos jerárquicos para 3 taxa de vertebrados e invertebrados de interés para la conservación (caracoles [Monadenia churchi], campañol rojo [Arborimus longicaudus], marta pescadora [Martes pennanti pacifica]) que proporcionan

Paper submitted August 27, 2009; revised manuscript accepted February 23, 2010. 
ejemplos de un rango de extensiones distributivas y habilidades disperoras. Utilizamos datos de presenciaausencia derivados de programas de monitoreos regionales para desarrollar modelos con covariables ambientales a nivel de paisaje y de sitio. Utilizamos algoritmos Monte Carlo cadena de Markov y un marco de modelo autoregresivo condicional o condicional intrínseco para ajustar los modelos espaciales. El ajuste de los modelos espaciales bayesianos fue entre 35\% y 55\% mejor que el ajuste de modelos análogos no espaciales. Los modelos espaciales Bayesiamos funcionaron mejor que modelos análogos desarrollados con métodos de entropía máxima (Entmax). Aunque los mejores modelos espaciales y no espaciales incluyeron variables ambientales similares, los modelos espaciales proporcionaron estimaciones de los efectos espaciales residuales que sugirieron cómo los procesos ecológicos pueden estructurar los patrones de distribución. Los modelos espaciales construidos con datos de presencia-ausencia mejoraron el ajuste para la mayoría de las espcies endémicas con rangos de distribución constreñidos por factores biogeográficos poco conocidos y para especies ampliamente distribuidas que se sospecha son afectadas fuertemente por variables ambientales no medidas o por procesos poblacionales. Al tratar los efectos espaciales como una variable de interés y no como una molestia, los modelos espaciales Bayesianos jerárquicos, especialmente cuando se basan en un entramado espacial de escala común (aquí hexágonos de $24 \mathrm{~km}^{2}$ del Inventario y Análisis Forestal nacional), pueden incementar la relevancia de los modelos de bábitat para la planificación de la conservación de múltiples especies.

Palabras Clave: autocorrelación espacial, autoregresivo condicional, dependencia espacial, especie focal, Martes pennanti, modelo autoregresivo espacial, modelo bayesiano jerárquico, modelo de distribución de especies, Plan Forestal Noroccidental

\section{Introduction}

Conservation planning and wildlife management increasingly draw on models of habitat relations derived from regional surveys of species occurrence to further understanding of a species' ecology and the factors limiting its distribution. Information from such models can also facilitate protection and enhancement of habitat, predict distribution in unsurveyed areas, and help evaluate suitability of currently unoccupied areas for reintroduction. Nevertheless, commonly used statistical techniques such as logistic regression may be poorly suited for developing geographically extensive distribution models due to their inadequate treatment of autocorrelation and other spatial aspects of the data (Dormann et al. 2007).

Spatial autocorrelation is a pervasive characteristic of data on species distributions because spatial autocorrelation of environmental factors and biological processes frequently results in aggregated distributions of individuals (Clark 2007). Spatial structure linked to environmental factors has been termed exogenous, induced, or extrinsic spatial effects. In contrast, endogenous, inherent, or intrinsic spatial effects arise from population processes such as dispersal and territoriality (Lichstein et al. 2002; Wintle \& Bardos 2006). Spatially autocorrelated data violate the assumption of independence in standard statistical tests, which can lead to inclusion of variables with spuriously significant parameters (Dormann et al. 2007). This reduces the interpretability of model structure and parameters and limits prediction accuracy when models are extrapolated to new regions or novel conditions, such as future climates. Rather than pursue spatial independence in survey data (e.g., through spatially dispersed measurements), it may be more effective to use alternative statistical methods that can partition the spatial component from the hypothesized environmental effects. This can increase the generality of the habitat relations described in the model and providing insights concerning the biological processes creating spatial dependence and the scales at which they operate (Bolker et al. 2009).

Faunal distribution models are commonly based on a form of generalized linear model (GLM) (Bolker et al. 2009). For example, logistic regression models a binary response (e.g., presence-absence) by means of a GLM with the logit link. Diverse approaches have been used to extend this approach to account for spatial autocorrelation. One group of methods incorporates spatial effects as additional covariates within the model (Beale et al. 2010). For example, trend-surface variables may be derived from geographic coordinates to model spatially extensive trends not explained by environment (Haining 2003). Simple autoregressive methods estimate spatial effect through an additional covariate on the basis of smoothed, observed occurrence values at neighboring sites within a spatial neighborhood (Beale et al. 2010). Because such models first estimate the spatial effect and then the environmental effects, spatial structure in the response data tends to be attributed to the spatial autocovariate and may thus underestimate the effect of environmental variables (Dormann 2007a). Beale et al. (2010) found that methods that model space as an additional covariate generally have poorer fit than those that model space in the error or random-effect term.

Hierarchical Bayesian autoregressive models take the approach of modeling space in the error term. These models simultaneously produce estimates of environmental variables and spatial random effects, which allows 
the data themselves to determine the best placement of spatial effects. This approach also allows better estimation where response data are missing than is possible with simple autoregressive methods (Gelman et al. 2004; Latimer et al. 2006). Hierarchical spatial autoregressive models, although usually intractable with standard statistical software, can be fit with Markov chain Monte Carlo (MCMC) techniques (Spiegelhalter et al. 2003). Non-Bayesian methods exist for the methods we considered but have not been widely tested (Bolker et al. 2009).

Bayesian spatial and nonspatial models built with artificial data sets (Wintle \& Bardos 2006; Dormann et al. 2007; Beale et al. 2010) or plant survey data (Latimer et al. 2006, 2009) have been compared previously. We (1) further developed and applied such techniques to a set of multispecies faunal survey data typical of regional planning efforts, (2) evaluated whether the use of spatial models alters model selection and prediction, (3) explored the contrasts between models using extensive covariates derived from geographic information systems (GIS) and those using covariates measured in sparsely distributed plots, and (4) developed and contrasted examples of models for vertebrate and invertebrate taxa with a range of dispersal abilities and territory sizes to assess whether the utility of spatial models varies across spatial scales.

\section{Methods}

\section{Survey Data}

We created and compared models for one invertebrate and two vertebrate species of conservation concern (Carroll et al. 1999; Dunk et al. 2004; Dunk \& Hawley 2009). Area-limited vertebrates with specialized resource requirements, such as the fisher (Martes pennanti pacifica), are a common focus of regional conservation planning and monitoring programs that collect extensive data on a particular species' occurrence (Carroll et al. 1999; Davis et al. 2007). Less commonly, monitoring focuses on a wider range of localized or poorly known taxa (Molina et al. 2006). Survey data were collected under the U.S. Northwest Forest Plan's Survey and Manage program (Molina et al. 2006) for two of the three species: Church's sideband snail (Monadenia churchi), which is an endemic mollusk, and the red tree vole (Arborimus longicaudus), which is a mammal with more extensive geographic range than the snail but with a similarly limited territory size and dispersal capability (Dunk et al. 2004; Dunk \& Hawley 2009). The three species (Fig. 1) span several orders of magnitude of maximum dispersal distance ( $<100 \mathrm{~m}$ for the snail to $>30 \mathrm{~km}$ for the fisher) and home range size (Dunk et al. 2004; Carroll et al. 1999).

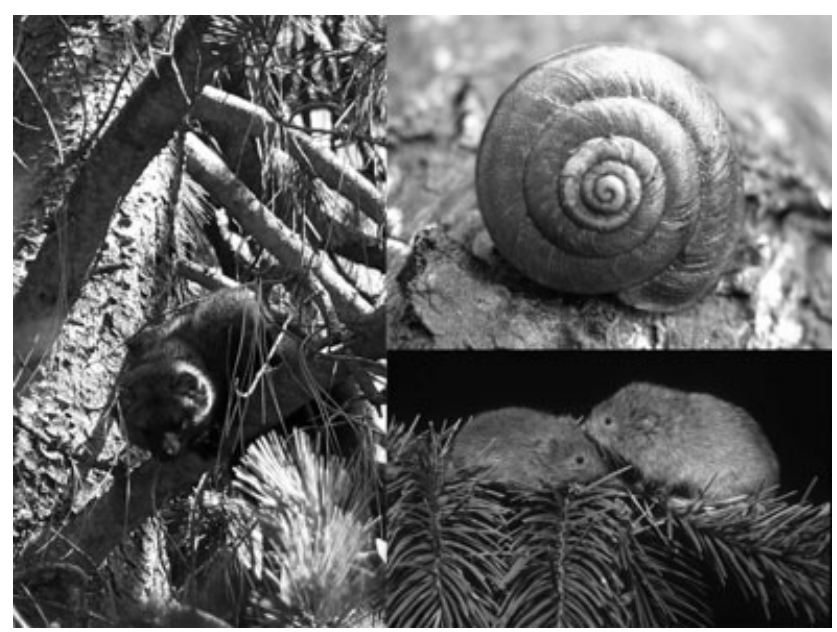

Figure 1. Three species found in montane forests of the Pacific Northwest and Sierra Nevada of the United States whose distributions are analyzed in this study (clockwise from left): Pacific fisher (Martes pennanti pacifica), Church's sideband snail (Monadenia churchi), and red tree vole (Arborimus longicaudus). Photo credits: Rebecca Green, Jeffrey Dunk, and Bert Gildart, respectively.

We obtained distributional data for the fisher, vole, and snail from previously conducted systematic presence-absence surveys (Dunk et al. 2004; Davis et al. 2007; Dunk \& Hawley 2009). Survey locations in those studies were based on the sampling scheme of the national Forest Inventory and Analysis (FIA) or Continuous Vegetation Survey (CVS) grid (Bechtold \& Patterson 2005). For the fisher, we also obtained additional data from previous nonsystematic surveys on private lands. The FIA program regularly collects standardized vegetation data at one 1-ha plot within each hexagon of a grid that has been delineated across the United States (see Fig. 3 for an example of plot distribution). The current (after 2001) FIA sampling grid contains hexagons of approximately $24 \mathrm{~km}^{2}$ (Bechtold \& Patterson 2005).

For species such as the vole and snail modeled relative to plot- or site-level covariates, we used surveys performed at FIA/CVS plots to allow access to vegetation data collected previously by the FIA program at these sampled locations and at other FIA/CVS sites to which we then could extrapolate the species distribution model. For species such as the fisher whose distribution was modeled as a function of covariates measured across the landscape, the FIA hexagonal grid provided a spatial lattice that facilitated parameterization of spatial neighborhood effects, reduced problems with uneven sampling intensity or survey effort, and reduced the number of sample units (to $<10,000$ ) so that spatial modeling at regional extents was computationally feasible. The size of FIA hexagons is biologically relevant to territory size for 
the fisher (10-30 $\mathrm{km}^{2}$ ) and the landscape extent with highest predictive power in previous analyses (Carroll et al. 1999). Although our surveys conformed to standard protocols designed to reduce the probability of false absence records, we did not have access to detection histories from repeated surveys, either because sites had been visited only once or data had been aggregated into a single presence-absence record per site. Therefore, we did not consider how detectability, as distinct from occurrence probability, varied across space and among species (Webster et al. 2008). Further description of survey data and protocols is given in Supporting Information.

\section{Autoregressive Modeling}

We applied two forms of spatial autoregressive models, depending on the spatial extent and resolution of the environmental covariates most relevant to the particular species. Our landscape- and site-level models thus differed in both their model structure and environmental covariates. We used landscape-level models to analyze the relationship between the fisher and environmental variables derived in a GIS. Distribution models for such wide-ranging species typically have the highest predictive power at or above the scale of the animal's territory (Carroll et al. 1999). Model predictions at this scale are also relevant to regional conservation planning efforts because they predict distribution seamlessly across the entire landscape.

We adapted the modeling framework of Latimer et al. (2006; their model 2). We overlaid a regular lattice of cells (the FIA hexagons) on the study area and considered each cell a sample unit that might contain a number of survey locations. In our model, $y_{i}$, the presence-absence at site $i$, was distributed as a binomial variable with probability $p_{i}=\exp \left\{\eta_{i}\right\} /\left(1+\exp \left\{\eta_{i}\right\}\right)$, where $\eta_{i}=\beta_{0}+$ $\beta_{1} x_{1 i}+\cdots+\beta_{p} x_{p i}+\rho_{i}$. The variables $x_{1 i}, \ldots, x_{p i}$ were site-specific environmental covariates, and the $\rho_{i}$ were random effects that were jointly distributed as a Gaussian CAR (conditional autoregressive) spatial model. The CAR model was defined by the conditional normal distributions $\rho_{i} \mid \rho_{-i} \sim N\left(\mu_{i}, \sigma_{i}^{2}\right)$, where $\rho_{-i}$ (spatial random effect) is the vector of all $\rho$ except the one for the $i$ th site:

$$
\mu_{i}=\frac{\phi}{n_{i}} \sum_{j \in N_{i}} \rho_{j} \quad \text { and } \quad \sigma_{i}^{2}=\frac{1}{\tau^{2} n_{i}},
$$

$n_{i}$ is the number of cells in the neighborhood, $N_{i}$ of the $i$ th cell, and $\tau$ represents the precision (inverse variance) parameter.

The weight specification $1 / n_{i}$ in $\mu_{i}$ and $\sigma_{i}^{2}$ is termed row standardized weighting (RSW) (see Supporting Information for the code used to produce weight matrix). The $\phi$ parameter dictates the amount of spatial correlation between spatial effects and lies in the interval $[0,1)$ for positive association. Typically values reside very near 1 . This observation motivated development of the intrinsic CAR (ICAR) model, a form of the CAR model in which $\phi=1$ (Banerjee et al. 2004). Although the ICAR conditional distributions do not specify a proper joint distribution (i.e., integrate to 1 ) for the $\rho$ vector, the parameter and prediction posterior distributions are proper (Banerjee et al. 2004). We compared ICAR models with first-order (a cell and its six immediate neighbors), second-order, and third-order neighborhoods.

Because models constructed with only landscape-level variables generally have poor fit and predictive power for localized low-vagility species, models for such species typically are constructed with site-level environmental variables (e.g., variables measured over a 1-ha FIA plot). We followed the framework of previous non-Bayesian habitat modeling for the vole and snail by building models from site-level environmental variables augmented with relevant spatially extensive (e.g., climate) covariates (Dunk et al. 2004; Dunk \& Hawley 2009). We analyzed these data with CAR models. Although in a strict sense, CAR and ICAR models are applied most appropriately to seamless lattices of cells, we applied the CAR framework to model site-level responses that resemble the point data typically analyzed with geostatistical models. A CAR model can closely approximate continuous geostatistical processes and is much more computationally efficient than a geostatistical model (Rue \& Held 2005; Webster et al. 2008; Ibánez et al. 2009), although recent development of "spatial predictive process" models may increase the computational feasibility of nonlattice-based approaches for large data sets (Finley et al. 2007; Latimer et al. 2009).

When environmental variables are measured at the level of sites, and sampled sites are sparse or unevenly distributed, distance between a site and its nearest neighbors may vary widely. This suggests that site-level data, in contrast to survey data summarized over larger sample units or cells, would benefit from the greater flexibility of a CAR model in which weights can vary with distance (Cressie 1993). We used CAR models with RSW, however, which approximates the weight structure of ICAR by assigning equal weights to all neighbors. In exploratory analyses of our data, RSW offered better model fit compared with more complex distance weighting schemes. Different weighting schemes can be implemented and compared with the code provided in Supporting Information. We specified the neighborhoods $N_{i}$ for the site-level data by defining a radius threshold of 6 , $8,14,20$, and $30 \mathrm{~km}$ centered around the $i$ th site. We considered all the other sites within these radius neighbors of the $i$ th site. The sampling design based on FIA plots separated by 5-6 km did not allow us to assess smaller spatial neighborhoods.

We used the program WinBUGS (version 1.4.3; Spiegelhalter et al. 2003) to fit ICAR models and 
their nonspatial generalized linear model analogues (see Supporting Information for BUGS code). We used OpenBUGS (version 3.0.3; Thomas et al. 2008) to fit CAR models and their nonspatial generalized linear model analogues. Both WinBUGS and OpenBUGS simulations used three MCMC chains, each with a burn-in period of 10,000 iterations followed by 40,000 iterations for estimation. We evaluated convergence with the Brooks-GelmanRubin statistic (Spiegelhalter et al. 2003). We assumed no preexisting knowledge of model parameters and thus specified the least informative priors that allowed model convergence (Supporting Information). To facilitate convergence of model estimates, we standardized variables by subtracting the mean and then dividing the mean by 2 SD (Gelman 2008).

\section{Descriptions of Candidate Models by Species}

We evaluated, through comparison with previously published results, whether the use of spatial models altered model selection and prediction. Therefore, rather than explore the set of all possible candidate models, we developed a set of models for each species on the basis of the best model identified in the publications from which the data set was drawn. We compared this model with alternate candidate models that we varied by assigning either linear or quadratic terms to each variable or dropping one or more variables. We thus evaluated whether addition of spatial random effects reduced the structure of the environmental component of the best model. We evaluated interaction terms only if they were present in the published best model. This resulted in 13-15 candidate models per species, depending on the number of variables in the published model (Supporting Information). We identified the best spatial models with a two-step process. First, we identified the best spatial neighborhood size (scale) by comparing mean squared predictive error (MSPE; see below) of the full model for a species across a range of scales. Second, we identified the best of the candidate models at the best scale.

For the snail, we developed 15 candidate models from the best model of Dunk et al. (2004), which included four covariates: mean diameter breast height (dbh) of conifers, percent canopy cover of hardwoods, coefficient of variation (cv) of December and July precipitation, and an index of moisture stress that related summer temperature and mean annual precipitation. For the vole, we developed 14 candidate models from the best model of Dunk and Hawley (2009), which included four independent variables: percent slope, basal area of trees with $\mathrm{dbh}$ between 45 and $90 \mathrm{~cm}$, maximum tree $\mathrm{dbh}$, and the standard deviation (SD) of conifer dbh. For the fisher, we built the candidate set of 13 models with seven variables identified in previous generalized linear or generalized additive habitat models (Carroll et al. 1999; Carroll et al. 2001; Davis et al. 2007): tree canopy closure, tree size class, annual snowfall, annual precipitation, terrain ruggedness, summer tasseled-cap greenness, which is a metric of primary productivity derived from satellite imagery, and a rating of fisher habitat quality derived from the California Wildlife Habitat Relationships system.

We did not consider spatial trend-surface variables (functions of the $x$ and $y$ coordinates) included in published models (Dunk et al. 2004; Dunk \& Hawley 2009) because they would duplicate trends modeled within the spatial random-effect term. Our nonspatial analogue models, which lacked both trend surfaces and spatial random effects, should thus be seen as "naïv" versions of published nonspatial models for these species. To better compare the performance of the CAR models with the most commonly used alternative methods for analyzing species' distributional data, we also developed distribution models for the three species with Maxent (Phillips et al. 2006; Phillips \& Dudik 2008). Maxent estimates probability distributions that are close to maximum entropy or uniform given constraints derived from the presence data and functions of the environmental variables (Phillips \& Dudik 2008). Maxent performs well in comparison with 15 alternate methods on a wide variety of taxa in diverse regions (Elith et al. 2006). Although Maxent typically is applied to presence-only data, it can be applied to presence-absence data, as we did here, by basing the set of available habitat on the total set of sampled locations (Phillips et al. 2009). We used the default Maxent setting, which allows flexible response forms such as thresholds and hinge effects (Phillips \& Dudik 2008). As with the GLM and CAR models, however, we evaluated in the Maxent analysis only those interaction effects present in published models. We also summarized results from previous studies of the three species with generalized additive models (GAM) that contained spatial covariates in the form of trend surfaces (Dunk et al. 2004; Dunk and Hawley 2009) or a simple autoregressive term (Davis et al. 2007).

\section{Comparison of Models}

We fitted candidate models and ranked competing models by their MSPE (Gelfand \& Ghosh 1998). Although deviance information criterion (DIC), a metric that identifies parsimonious models via a penalty term derived from the number of model parameters, is the most common model diagnostic used to evaluate fit of Bayesian models, its utility depends on the accuracy of its estimation of the effective number of parameters in the model $\left(p_{D}\right)$ (Spiegelhalter et al. 2002; Gelman et al. 2004). In cases in which DIC's normality assumptions are violated, $p_{D}$ may be estimated as negative; thus, the DICbased model ranking is biased. In contrast, MSPE lacks such assumptions. The MSPE evaluates a model on the basis of its ability to accurately produce replicated data similar to the data that were observed (Gelman et al. 
2004). Because more-complex models tend to produce replicated data slightly more accurately, MSPE tends to choose slightly more complex models than does DIC and alternate, closely competing models are less differentiated. Unlike DIC, MSPE does not explicitly penalize an increasing number of model parameters. Nevertheless, as model complexity becomes too great, the variability in the replicated data becomes considerable which, in turn, raises MSPE and reduces model rank. Thus MSPE and DICbased model rankings are often similar (Carroll \& Johnson 2008).

Diagnostic metrics also have been developed for Bayesian models, and they are based on comparisons of observed responses with simulations of predicted responses (Gelman et al. 2004). We used the posterior predictive $p$ value to evaluate the probability that the observed and the predicted data were drawn from the same distribution (Gelman et al. 2004). Similarly, we produced a percent correct classification metric that we based on the proportion of simulated responses matching observed responses. We evaluated the difference between spatial and nonspatial models with Spearman rank correlation between predictions from the best spatial model and its nonspatial analogue (corrs) and the percent reduction in MSPE from nonspatial to spatial models.

For spatial, nonspatial, and Maxent models, we evaluated the AUC (area under the receiver-operating curve), a threshold-independent metric used to evaluate a model's discriminatory ability (Swets 1988). The AUC ranges between 0.5 (for a model with performance equal to random expectation) and 1.0 (for a model with perfect classification). To evaluate the potential for overfitting and the sensitivity of model results to subsetting of the input data, we used a cross-validation procedure that randomly withheld $10 \%$ of the samples and evaluated the ability of models developed from the remaining samples to predict the data that were withheld. We repeated cross-validation 10 times for the best model for each species and reported the mean of the resultant AUC values.

We compared general patterns in model results across species, including the size of the spatial neighborhood associated with the best model. We evaluated the statistical and ecological implications of differences among the species in correlation between predictions of spatial and nonspatial models. We evaluated the increase in fit of the model (proportional reduction in MSPE) between nonspatial and spatial models. For each species, we summarized the structure of the best spatial and nonspatial models. We described the spatial pattern of the randomeffect term. For the species modeled at the landscape level, we described the spatial pattern of predicted probability on the basis of environmental effects alone (without the $\rho$ term). Evaluating predicted abundance without the $\rho$ term allowed us to assess the effects of predicted species-environment relationships without the influence of unmeasured environmental variables.

\section{Results}

\section{General Patterns}

Across all species, models based on the smallest spatial neighborhoods generally fit best (Fig. 2). Models based on neighborhoods with an $8-\mathrm{km}$ radius, the smallest evaluated at the landscape level, performed best for the fisher. Models based on neighborhoods with a $6-\mathrm{km}$ radius, the smallest evaluated in the site-level models, fit best for the snail, whereas models with an $8-\mathrm{km}$ neighborhood radius fit best for the vole (Fig. 2).

Spatial models had better fit than nonspatial models for all species (Table 1). The improvement in fit conferred by the spatial model, as measured by the percent reduction in MSPE from nonspatial to spatial models, was $35.3,54.7$, and 54.0 for the vole, the fisher, and the snail, respectively (Fig. 2, Table 1). The correlation between spatial and nonspatial model predictions generally paralleled the difference in fit between spatial and nonspatial models (Table 1). Percent correct classification improved from $70.4,84.8$, and $73.6 \%$ (nonspatial models) to 81.3 , 91.8 , and $88.3 \%$ (spatial models) for the vole, the fisher, and the snail, respectively.

For all species, for both the full data set and crossvalidation runs, AUC values for the spatial models were greater than AUC for the nonspatial or Maxent models (Table 1). Although posterior predictive $p$ value for both spatial and nonspatial models for all species suggested that predicted and observed values were drawn for the same distribution (i.e., the model was not over-fit) (Table 1), contrast between AUC and CV AUC was greater for spatial than for nonspatial models. Thus, the improvement in AUC associated with use of spatial models was

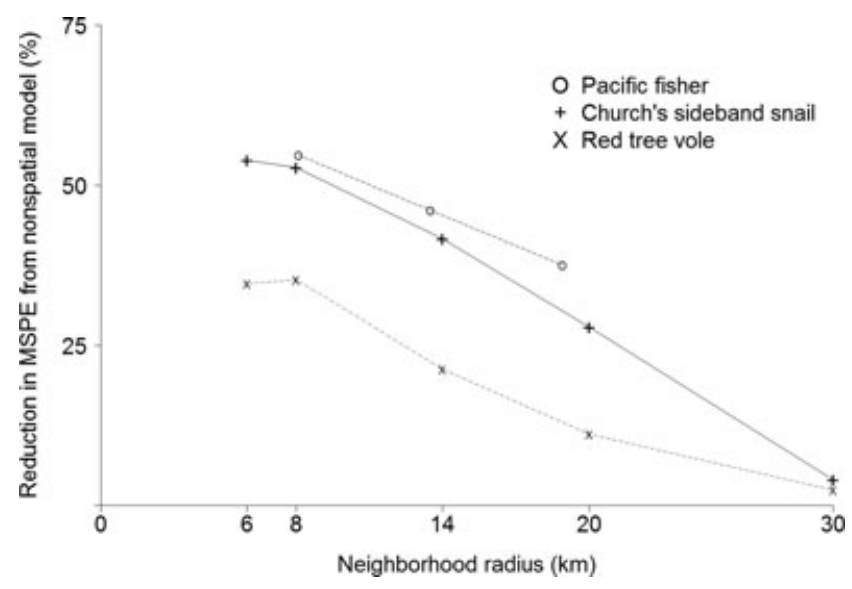

Figure 2. Improvement in model fit, as measured by percent reduction in the MSPE (mean square predictive error) diagnostic, of spatial models over their nonspatial analogues for three species. Curves are left-truncated at $6 \mathrm{~km}$, the approximate mean distance between survey locations. 
Table 1. Comparison across species of model performance and difference between spatial and nonspatial models for red tree vole, Pacific fisher, and Church's sideband snail. ${ }^{a}$

\begin{tabular}{lccc}
\hline & \multicolumn{3}{c}{ Species } \\
\cline { 2 - 4 } & $\begin{array}{c}\text { red } \\
\text { tree } \\
\text { vole }\end{array}$ & $\begin{array}{c}\text { Pacific } \\
\text { fisher }\end{array}$ & $\begin{array}{c}\text { Church's } \\
\text { sideband } \\
\text { snail }\end{array}$ \\
\hline Model performance & & & \\
nonspatial GLM & & & \\
PCC & 0.704 & 0.848 & 0.736 \\
PPPV & 0.432 & 0.494 & 0.448 \\
spatial CAR & & & \\
PCC & 0.813 & 0.918 & 0.883 \\
PPPV & 0.529 & 0.558 & 0.518 \\
correlation & 0.843 & 0.596 & 0.528 \\
MSPE reduction & 35.26 & 54.70 & 53.95 \\
Comparison of AUC & & & \\
among methods & & & \\
nonspatial GLM & & & \\
full & 0.814 & 0.720 & 0.746 \\
CV & 0.783 & 0.724 & 0.690 \\
spatial CAR & & & \\
full & 0.985 & 0.940 & 0.994 \\
CV & 0.807 & 0.851 & 0.863 \\
maximum entropy (Maxent) & & & \\
full & 0.852 & 0.769 & 0.832 \\
CV & 0.783 & 0.741 & 0.774 \\
GAM with spatial term & & & \\
full & $0.866^{b}$ & $0.96^{c}$ & $0.844^{d}$ \\
CV & $0.798^{b}$ & $0.86^{c}$ & $0.795^{d}$ \\
\hline
\end{tabular}

${ }^{a}$ The difference between spatial and nonspatial models was evaluated with PCC (proportion correctly classified) and PPPV (posterior predictive p value) statistics, the Spearman rank correlation between predictions from the best spatial model and its nonspatial analogue, and the percent reduction in MSPE (mean squared predictive error) from nonspatial to spatial models. Model performance was also evaluated with the AUC (area under the receiver operating characteristic curve) metric for the full data set and for data withbeld during 10 fold cross-validation for nonspatial GLM (generalized linear model), spatial CAR (conditional autorgressive model), and Maxent models and was compared with AUC reported in previous studies in which GAM (generalized additive model) with spatial components was used. Number of sampled sites or hexagons, $n=365,993$, and 308 for red tree vole (A. longicaudus), Pacific fisher (M. p. pacifica), and Church's sideband snail (M. churchi), respectively.

${ }^{b}$ Dunk and Hawley 2009.

${ }^{c}$ Davis et al. 2007.

${ }^{d}$ Dunk et al. 2004

less pronounced under the cross-validation runs than with the full data set. In Maxent models, AUC was intermediate between nonspatial and spatial models for all species under both full and cross-validation runs.

\section{Summary of Models}

For the snail, a model with linear terms for three of four variables (mean dbh of conifers, cr of December and July precipitation, and moisture stress) and a quadratic term for the remaining variable (percent canopy cover of hardwoods) was the best spatial model. A model with quadratic terms for three variables (mean dbh of conifers, percent canopy cover of hardwoods, moisture stress) and a linear term for $\mathrm{cv}$ of December and July precipitation was the best nonspatial model (Supporting Information). For the vole, a model with quadratic terms for all four variables (percent slope, basal area of trees, maximum tree $\mathrm{dbh}, \mathrm{SD}$ of conifer $\mathrm{dbh}$ ) had the lowest MSPE among the candidate nonspatial models. The best spatial model was similar to the best nonspatial model except with a linear term on one variable (maximum tree $\mathrm{dbh}$ ). For the fisher, the best spatial model contained linear terms for the variables tree canopy closure, greenness, and terrain ruggedness and a quadratic term for annual snowfall. The best nonspatial model was similar to the best spatial model, but it had a linear term for snowfall. Generally, spatial models and their nonspatial analogues had similar ranks (Supporting Information).

The broad-scale pattern of spatial random effects was relatively smooth (low rugosity) for the snail and the fisher (Figs. 3a \& 4a). Nevertheless, spatial random effects were concentrated in a single population for the snail and in two disjunct populations for the fisher. Greater mesoscale variation in spatial random effects was evident for the vole (Fig. 3b). For the fisher, the environmental component from the selected model (without the spatial random effects) predicted high habitat quality for a large area in the northern Sierra Nevada (Fig. 4b).

\section{Discussion}

Biologists and managers who develop and apply habitat models are often familiar with the statistical challenges posed by their data's spatial structure, but are unsure whether more complex spatial models will increase the utility of model results in planning (Beale et al. 2010). We analyzed presence-absence data for three species with different dispersal abilities and home range sizes, using environmental variables measured at the level of both landscapes and sites. Thus, our conclusions may generalize across the spectrum of potential applications of spatial models of habitat for faunal species and should assist in deciding when to use spatial models, choosing the appropriate model structure, and interpreting model results.

Our results suggest that the computational time and effort required to develop spatial models is often worthwhile because spatial models fit observed data better than nonspatial models and allow greater insights than nonspatial models into the spatial processes producing autocorrelation in the data. For all species, for both the full data set and cross-validation runs, model performance as measured by the AUC value was greater for spatial CAR models than for nonspatial logistic regression or maximum entropy (Maxent) models. Nevertheless, differences between model performance under full and cross-validation runs were greater for spatial than for nonspatial models, which suggests that results of spatial models may be 


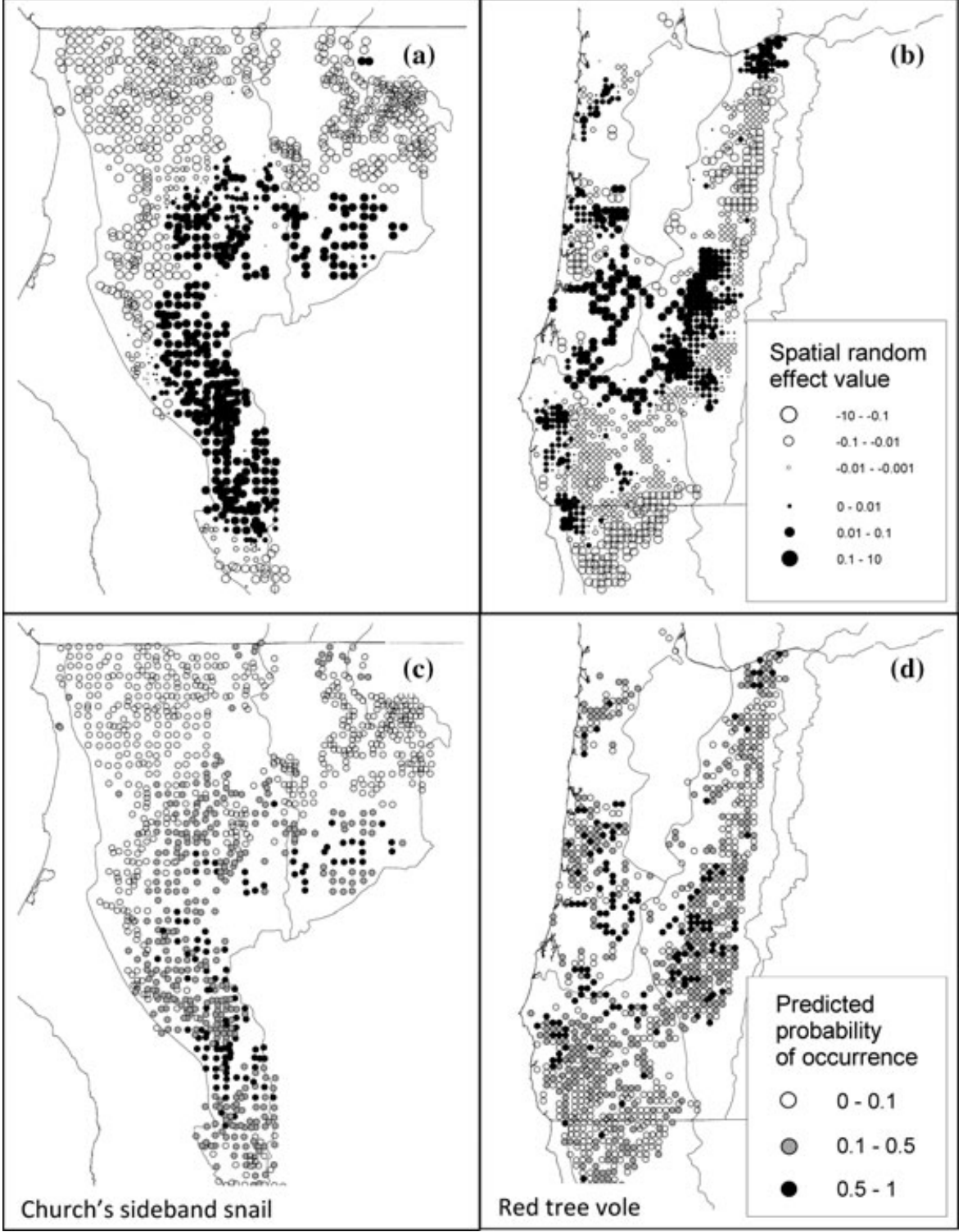

Figure 3. Pattern of $(a, b)$ spatial random effect and $(c, d)$ predicted probability of occurrence from best spatial conditional autogressive (CAR) models for Church's sideband snail and red tree vole modeled with site-level covariates in California and Oregon (U.S.A.). moderately sensitive to variation in input data (e.g., due to unsurveyed portions of a region). Maxent models had similar sensitivity to data subsetting.

The performance of spatial models for the vole and the snail was better than that of previous spatial GAM models for those species (Table 1). Nevertheless, our spatial model for the fisher had slightly worse performance than a GAM model from a previous study that incorporated a simple autoregressive spatial term derived from the smoothing of observed responses (Davis et al.

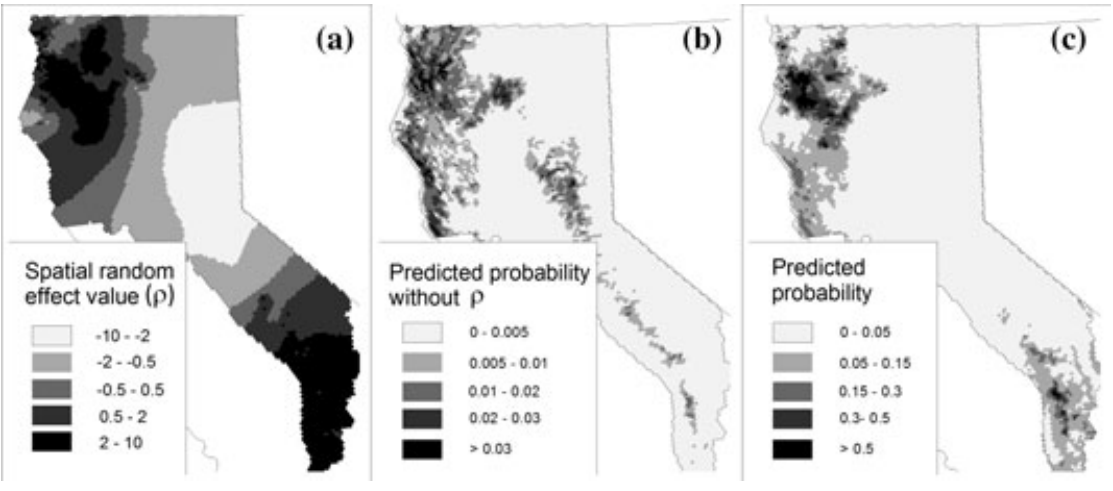

Figure 4. Pattern of (a) spatial random effect, (b) predicted probability of occurrence without the spatial random-effect term, and (c) predicted probability from best spatial intrinsic conditional autogressive (ICAR) model for the Pacific fisher, a wide-ranging species, in the California (U.S.A.). Probability of occurrence was modeled as a function of landscape-level covariates. 
2007). Davis et al. (2007) constrained the simple autoregressive term to enter the model after the best environmental variables had been identified, and used slightly different fisher distribution data and cross-validation procedure than our study. Our results are consistent with those from a recent review whose authors compared methods using simulated spatially correlated data. The review showed that methods with spatial effects in the error term, such as the CAR and ICAR methods, generally performed better than models with spatial effect as an additional covariate (such as spatial trend surface or simple autoregressive methods) (Beale et al. 2010). GAM performed best among the latter methods, however, and both groups of spatial methods outperformed nonspatial approaches (Beale et al. 2010).

Lack of explicit treatment of spatial structure may lead to inclusion in the model of spurious environmental variables (Dormann et al. 2007; Dormann 2007b). In our results, the difference between the fit of analogous spatial and nonspatial models lessened as models become more complex (Supporting Information), which suggests that a subset of the environmental covariates may be acting as surrogate for spatial structure in the nonspatial models. Our results also provide insights into the scale at which spatial processes influence distribution (Fig. 2). For the two species for which data allowed comparison of 6-and 8-km neighborhoods, the best models for the snail with low dispersal ability were at a smaller neighborhood size than the best models for the more vagile vole. Although our results for the snail and the fisher allow for the possibility that finer-resolution sampling designs would support improved models, in regional monitoring programs with limited resources the benefits of increased sampling intensity must be balanced against decreases in the extent of the region sampled. Our ICAR model results are consistent with the results of previous studies, which found first-order neighborhoods are often sufficient to account for spatial structure in distribution data (Griffith 1996; Kissling \& Carl 2008).

The high rugosity of the spatial effect in models for the vole (Fig. 3b) may have been due to mesoscale processes such as dispersal limitation rather than spatially extensive environmental effects. In contrast, the smoothness of the pattern of spatial effect for the fisher (Fig. 4a) may be due to unmeasured environmental variables or population processes, but we cannot conclusively distinguish between these hypotheses. The pattern of spatial effect for the fisher (Fig. 4a) closely resembles that of a sympatric raptor of conservation concern, the California Spotted Owl (Strix occidentalis occidentalis), which suggests the species are responding to a common unmeasured covariate (C. C., unpublished data).

Evaluating predicted occurrence without the $\rho$ term allowed us to assess the effects of predicted species-environment relationships without the influence of unmeasured environmental variables or population processes. The environmental component of the best model for the fisher identified a large area in the northern Sierra Nevada as fisher habitat (Fig. 4b). If the spatial random effects for the fisher can be attributed to population processes (home range clustering or dispersal limitation), this area may be a potential reintroduction site.

\section{Extracting Ecological Hypotheses from Spatial Structure}

Dormann (2007b) found that adding spatial effect to distribution models improved model fit in most contexts. Our results suggest that spatial models are most useful when either results of previous studies suggest strong spatial patterns in residuals from nonspatial models, as for the fisher (Carroll et al. 1999; Davis et al. 2007), or a species occupies a small proportion of the analysis region and its range boundary is complex, poorly known, or potentially constrained by past geologic or climatic events, as for the mollusk (Dunk et al. 2004). Spatial models are less useful when the analysis, survey extent, or survey data have been limited a priori to an area of relatively uniform spatial random effects, such as a well-described and cohesive range boundary.

Our results suggest that despite their greater computational cost, Bayesian spatial models perform better than other approaches to spatial modeling. This was most evident when we compare Bayesian methods to nonautoregressive approaches, such as trend-surface models. Although trend-surface variables can represent broadscale spatial trends, their flexibility is limited to quadratic or occasionally cubic surfaces. Thus, they cannot represent complex spatial-effect surfaces such as were shown in our analyses of the vole (Fig. 3c). More complex CAR models derived from distance weighting can also be used when they improve fit. In addition, CAR models, unlike trend-surface or generalized additive models, allow quantitative estimates of the strength of the spatial association with a single parameter $(\tau)$. Although the survey data we used were collected with a systematic sampling design, spatial autoregressive models may also aid analyses of data with geographically uneven levels of survey effort because such bias can be incorporated within the spatial random-effect term, which reduces its influence on estimates of the effects of environmental variables (Carroll \& Johnson 2008).

By treating spatial effects as a variable of interest rather than a nuisance, hierarchical Bayesian spatial models can suggest the identity of additional environmental covariates that may improve model fit or the existence of area- and isolation effects that may limit population viability. Our results suggest that spatial models developed at coarse resolutions (such as FIA hexagons) are statistically and biologically relevant to processes influencing the distribution of diverse taxa. Standardizing modeling efforts for multiple taxa to a common spatial 
lattice can help link regional-scale monitoring efforts and increase the relevance of habitat models to multispecies conservation planning.

\section{Acknowledgments}

We thank the many biologists who assisted in collection of species distribution data. The U.S. Department of Agriculture Forest Service Pacific Southwest Region, Pacific Northwest Region Survey and Manage Program, and the U.S. Department of Interior Fish and Wildlife Service provided funding for the surveys. T. Martin and two anonymous reviewers provided helpful comments.

\section{Supporting Information}

Description of survey data (Appendix S1), results of comparisons of models (Appendix S2), and example code for ICAR and CAR models (Appendix S3) are available as part of the online article. The author is responsible for the content and functionality of these materials. Queries (other than absence of the material) should be directed to the corresponding author.

\section{Literature Cited}

Banerjee, S., B. P. Carlin, and A. E. Gelfand. 2004. Hierarchical modeling and analysis for spatial data. Chapman \& Hall/CRC, Boca Raton, Florida.

Beale, C. M., J. J. Lennon, J. M. Yearsley, M. J. Brewer, and D. A. Elston. 2010. Regression analysis of spatial data. Ecology Letters 13:246-264.

Bechtold, W. A., and P. L. Patterson (editors). 2005. The enhanced forest inventory and analysis program-national sampling design and estimation procedures. General technical report SRS-80. U.S. Department of Agriculture Forest Service Southern Research Station, Asheville, North Carolina.

Bolker, B. M., M. E. Brooks, C. J. Clark, S. W. Geange, J. R. Poulsen, M. H. H. Stevens, and J.-S. S. White. 2009. Generalized linear mixed models: a practical guide for ecology and evolution. Trends in Ecology \& Evolution 24:127-135.

Carroll, C. and D. S. Johnson. 2008. The importance of being spatial (and reserved): assessing Northern Spotted Owl habitat relationships with hierarchical Bayesian models. Conservation Biology 22:1026-1036.

Carroll, C., R. F. Noss, and P. C. Paquet. 2001. Carnivores as focal species for conservation planning in the Rocky Mountain region. Ecological Applications 11:961-980.

Carroll, C., W. J. Zielinski, and R. F. Noss. 1999. Using survey data to build and test spatial habitat models for the fisher (Martes pennanti) in the Klamath region, U.S.A. Conservation Biology 13:1344-1359.

Clark, J. S. 2007. Models for ecological data: an introduction. Princeton University Press, Princeton, New Jersey.

Cressie, N. A. C. 1993. Statistics for spatial data. John Wiley and Sons, New York.

Davis, F. W., C. Seo, and W. J. Zielinski. 2007. Regional variation in home-range-scale habitat models for fisher (Martes pennanti) in California. Ecological Applications 17:2195-2213.

Dormann, C. F., et al. 2007. Methods to account for spatial autocorrelation in the analysis of species distributional data: a review. Ecography 30:609-628.
Dormann, C. F. 2007a. Assessing the validity of autologistic regression. Ecological Modelling 207:234-242.

Dormann, C. F. 2007b. Effects of incorporating spatial autocorrelation into the analysis of species distribution data. Global Ecology and Biogeography 16:129-138.

Dunk, J. R., and J. V. G. Hawley. 2009. Red-tree vole habitat suitability modeling: implications for conservation and management. Forest Ecology and Management 258:626-634.

Dunk, J. R., W. J. Zielinski, and H. K. Preisler. 2004. Predicting the occurrence of rare mollusks in northern California forests. Ecological Applications 14:713-729.

Elith, J., et al. 2006. Novel methods improve prediction of species' distributions from occurrence data. Ecography 29:129-151.

Finley, A. O., S. Banerjee, and B. P Carlin. 2007. spBayes: an R package for univariate and multivariate hierarchical point-referenced spatial models. Journal of Statistical Software 19(4):1-24.

Gelfand, A. E., and S. K. Ghosh. 1998. Model choice: a minimum posterior predictive loss approach. Biometrika 85:1-11.

Gelman, A. 2008. Scaling regression inputs by dividing by two standard deviations. Statistics in Medicine 27:2865-2873.

Gelman, A., J. B. Carlin, H. S. Stern, and D. B. Rubin. 2004. Bayesian data analysis. 2nd edition. Chapman \& Hall/CRC Press, Boca Raton, Florida.

Griffith, D. A. 1996. Some guidelines for specifying the geographic weights matrix contained in spatial statistical models. Pages 65-82 in S. L. Arlinghaus, editor. Practical handbook of spatial statistics. CRC Press, Boca Raton, FL.

Haining, R. 2003. Spatial data analysis: theory and practice. Cambridge University Press, Cambridge, United Kingdom.

Ibánez, I., J. A. Silander, Jr, A. M. Wilson, N. Lafleur, N. Tanaka, and I. Tsuyama. 2009. Multivariate forecasts of potential distributions of invasive plant species. Ecological Applications, 19:359-375.

Kissling, W.D., and G. Carl. 2008. Spatial autocorrelation and the selection of simultaneous autoregressive models. Global Ecology and Biogeography 17:59-71.

Latimer, A. M., S. Banerjee, H. Sang, E. S. Mosher, and J. A. Silander Jr. 2009. Hierarchical models facilitate spatial analysis of large data sets: a case study on invasive plant species in the northeastern United States. Ecology Letters 12:144-154.

Latimer, A. M., S. Wu, A. E. Gelfand, and J. A. Silander Jr. 2006. Building statistical models to analyze species distributions. Ecological Applications 16:33-50.

Lichstein, J.W., T. R. Simons, S. A. Shriner, and K. E. Franzreb. 2002. Spatial autocorrelation and autoregressive models in ecology. Ecological Monographs 72:445-463.

Molina, R., B. G. Marcot, and R. Lesher. 2006. Protecting rare, oldgrowth, forest-associated species under the survey and manage program guidelines of the Northwest Forest Plan. Conservation Biology 20:306-318.

Phillips, S. J., R. P. Anderson, and R. E. Schapire. 2006. Maximum entropy modeling of species geographic distributions. Ecological Modeling 190:231-259.

Phillips, S. J., and M. Dudik. 2008. Modeling of species distributions with Maxent: new extensions and a comprehensive evaluation. Ecography 31:161-175.

Rue, H. and L. Held. 2005. Gaussian Markov random fields: theory and applications. Chapman \& Hall/CRC, Boca Raton, FL.

Spiegelhalter D. J., N. G. Best, B. P. Carlin and A. Van Der Linde. 2002. Bayesian measures of model complexity and fit (with discussion). Journal Royal Statististical Society B 64:583640.

Spiegelhalter, D., A. Thomas, N. Best, and D. Lunn. 2003. WinBUGS user manual. Version 1.4. MRC Biostatistics Unit, Cambridge, United Kingdom. Available from http://www.mrc-bsu.cam.ac.uk/bugs (accessed November 2008).

Swets, J. A. 1988. Measuring the accuracy of diagnostic systems. Science 240:1285-1293. 
Thomas, A., B. O'Hara, U. Ligges, and S. Sturtz. 2008. Making BUGS Open. R News 6: 12-17. Available from http://mathstat. helsinki.fi/openbugs/ (accessed November 2008).

Webster, R. A., K. H. Pollock, and T. R. Simons. 2008. Bayesian spatial modeling of point transect data from bird surveys. Journal of Agricultural, Biological and Environmental Statistics 13:121139.

Wintle, B. A., and D. C. Bardos. 2006. Modeling species-habitat relationships with spatially autocorrelated observation data. Ecological Applications 16:1945-1958.

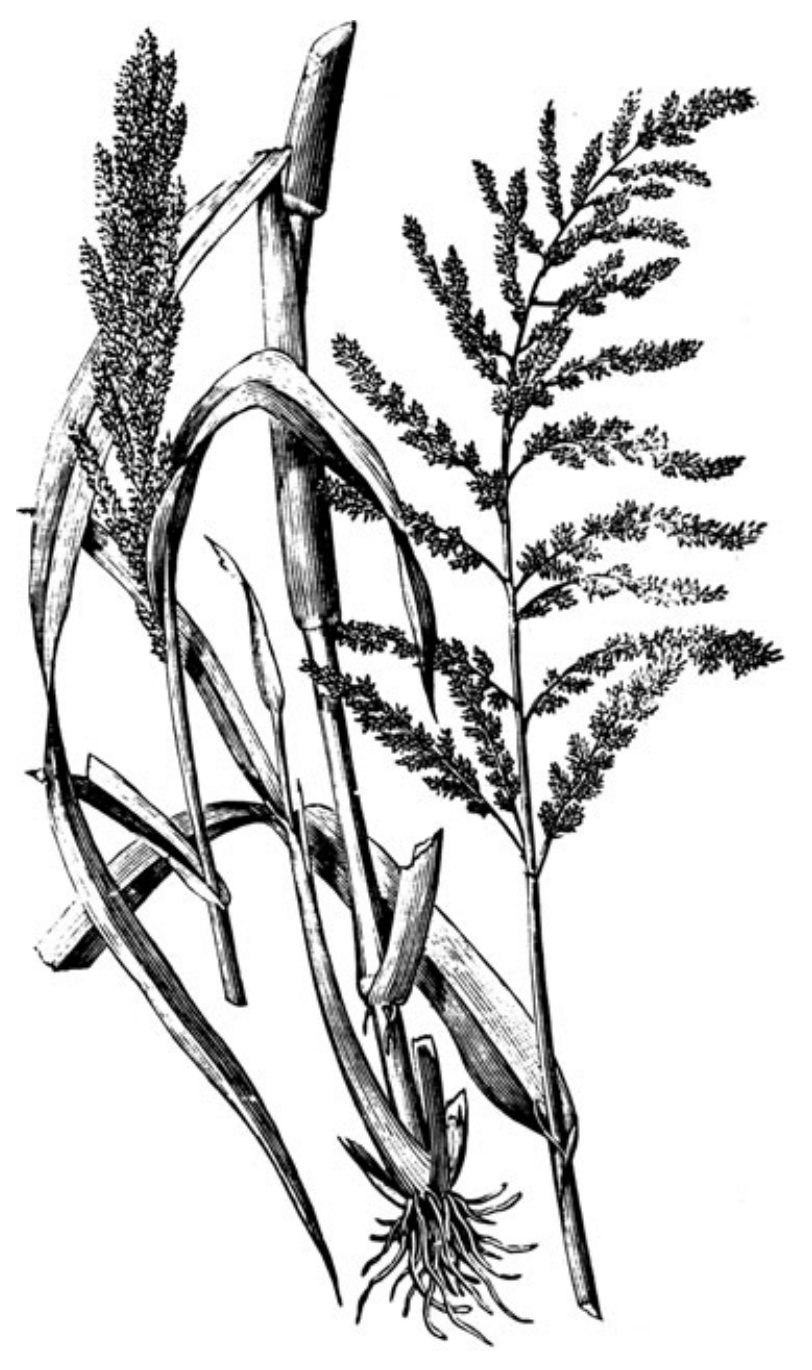

